\title{
Effect of inhibition of glycogen synthase kinase-3 on cardiac hypertrophy during acute pressure overload
}

\author{
Fumio Yamamoto, MD · Hiroshi Yamamoto, MD
}

Received: 30 August 2009

(C) The Japanese Association for Thoracic Surgery 2010

\begin{abstract}
Myocardial hypertrophy has been recognized to be an adaptive response to a variety of external stimuli (e.g., myocardial infarction, pressure overload, catecholamine treatment, endocrine disorders) that are involved in several subcellular factors that mediate signaling pathways, from external stimuli to nuclear protein syn-

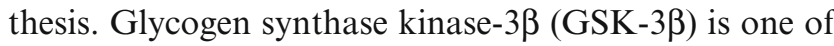
the subcellular factors that regulate nuclear transcription factors, such as activated T-cell (NFAT) proteins, that are related to gene programming during cardiac hypertrophy. On the other hand, GSK-3 $\beta$, known as a regulator of cardiomyocyte growth in Wnt signaling of cardiogenesis, is involved in $\beta$-catenin degradation. Inhibition of GSK-3 $\beta$ has been reported to induce cardiac hypertrophy. Tateishi et al. demonstrated in an aortic constriction-induced acute hypertrophy model using 6-week-old Wister rats that if GSK-3b is inhibited by $\mathrm{LiCl}$ up-regulated $\beta$-catenin expression and additional hypertrophy were observed. They suggested that $\mathrm{Li}^{2+}$ had an additive effect on pressure overload-induced hypertrophy through the GSK-3 $\beta$ - $\beta$-catenin pathway. Their article provides promising information on the mechanism of hypertrophic myocyte growth during acute pressure overload.
\end{abstract}

This editorial refers to the article by Tateishi et al. on pp 265-270 of this issue of General Thoracic and Cardiovascular Surgery.

F. Yamamoto $(\bowtie) \cdot H$. Yamamoto

Department of Cardiovascular Surgery, Akita University School of Medicine, Hondo 1-1-1, Akita, Akita 010-8543, Japan

Tel. +81-18-884-6135; Fax +81-18-836-2625

e-mail: f-yama@cvs.med.akita-u.ac.jp

\section{Introduction}

Myocardial hypertrophy has been recognized to be an adaptive response to a variety of external stimuli such as myocardial infarction, pressure overload, catecholamine treatment, and endocrine disorders, which are involved in several subcellular factors that mediate signaling pathways, from external stimuli to nuclear protein synthesis. Intracellular signaling pathways contributing to cardiac hypertrophy have been reported to include G-protein isoforms ( $\mathrm{Gi}$ and $\mathrm{Gq}$ ), low-molecular-weight guanosine transpeptidases (Ras, RhoA, Rac), calcineurin (calcium/calmodulin-dependent protein phosphatase), protein kinase $\mathrm{C}, \mathrm{p} 38$ mitogen-activated protein kinase (MAPK) isoforms, insulin-like growth factor I receptor, gp130-leukemia inhibitor factor receptor (LIFR) complex, basic fibroblast growth factor (FGF), transforming growth factor- $\beta$ (TGF $\beta$ ), and many others. ${ }^{1}$

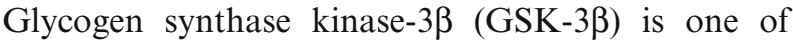
the subcellular factors regulating nuclear transcription factors such as activated T cell (NFAT) proteins, which are related to gene programming during cardiac hypertrophy. ${ }^{1,2}$ If NFAT proteins are dephosphorylated by calcineurin, the dephosphorylated NFAT proteins are translocated from the cytosol to the nucleus (i.e., nuclear import), resulting in cardiac hypertrophy. In contrast, if NFAT proteins are phosphorylated by GSK-3 $\beta$, the phosphorylated NFAT proteins are translocated in the reverse direction (i.e., nuclear export), antagonizing the calcineurin effect. In the unstimulated cell GSK-3 $\beta$ is in the active state, inhibiting the hypertrophic gene program, whereas in the stimulated cell GSK-3 $\beta$ is inactivated, NFAT proteins are dephosphorylated and translocated into the nucleus, and subsequently the hypertrophic gene program is promoted. In the 
transgenic mice overexpressing GSK-3 $\beta$, the heart weight/body weight ratio has been reported to be less than that in wild-type mice. ${ }^{3}$ A variety of external stimuli such as isoproterenol (ISO), endothelin-1 (ET-1), and ventricular pressure overload are known to inhibit the activity of GSK-3 $\beta$, inducing cardiac hypertrophy. Cyclosporin A, a calcineurin inhibitor, has been reported to counteract the effect of angiotensin II (Ang II), ET-1, or phenylephrine (PE) on inducing cardiac hypertrophy, suggesting that a calcium/calmodulin-dependent pathway through calcineurin may play an important role in the development of Ang II-, ET-1-, and PE-induced cardiac hypertrophy. ${ }^{2}$ FK506 has been demonstrated to prevent pressure overload-induced cardiac hypertrophy by inhibiting calcineurin activity and its gene expression. ${ }^{4}$

On the other hand, GSK-3 $\beta$ plays an important role in the regulation of cardiomyocyte growth in Wnt signaling of cardiogenesis during embryonic development. ${ }^{5}$ A GSK-3 $\beta$ molecule is coupled to a scaffolding protein (Axin) and an adenomatous polyposis coli (APC) protein, as well as phosphorylates $\beta$-catenin, resulting in ubiquitination and degradation of $\beta$-catenin. If GSK-3 $\beta$ activity is inhibited by its serine- 9 residue phosphorylation, $\beta$-catenin is stabilized and translocated to the nucleus to promote a cardiogenic gene program. Haq et al. have reported that $\beta$-catenin can be stabilized after being exposed to external stimuli (PE, ET-1, LiCl, pressure overload) ${ }^{6,7}$ in terminally differentiated cells, the mechanism of which is different from Wnt signaling. ${ }^{8}$ This study suggests a possibility that $\beta$-catenin stabilization may be involved in the cardiac hypertrophic response in mature myocardium through the pathways of GSK-3 $\beta$ inhibition.

The article from Tateishi et al. demonstrated in an aortic constriction-induced acute hypertrophy model using a 6-week-old Wister rat that if GSK-3b is inhibited by $\mathrm{LiCl}$ up-regulated $\beta$-catenin expression and additional hypertrophy were observed, and suggested that $\mathrm{Li}^{2+}$ had an additive effect on pressure overload-induced hypertrophy through the GSK-3 $\beta$ - $\beta$-catenin pathway.

In this study, there seemed to be three possibilities in terms of the mechanism of the additive hypertrophy induced with combined treatment of 2-day aortic banding and $\mathrm{LiCl}$ injection in a 6 -week-old rat. First, the extent of pressure overload might be insufficient for inhibition of GSK-3 $\beta$ activity and subsequent stabilization of $\beta$-catenin, and if so assessment in a rat with more severe aortic banding might provide useful information on the relative contribution of pressure overload to the development of hypertrophy. Second, a relatively short period (i.e., 2 days) of pressure overload might result in modest inhibition of GSK-3 $\beta$ activity, which might not be long enough to induce sufficiently $\beta$-catenin stabilization and resultant cardiac hypertrophy, requiring the adjuvant effect of $\mathrm{Li}^{2+}$. Third, the inhibiting effect of $\mathrm{Li}^{2+}$ on GSK-3 $\beta$ activity might induce hypertrophy additively through the other pathways such as calcineurin-NFAT signaling. The involvement of calcineurin-NFAT signaling in the additive effect of $\mathrm{Li}^{2+}$ might not be able to be ignored, although its contribution to the hypertrophy gene program has been assessed in rats with a longer exposure of pressure overload (e.g., 3 weeks of aortic banding).

In conclusion, the article from Tateishi et al. provides promising information on the mechanism of hypertrophic myocyte growth during acute pressure overload. Further investigation may be required in terms of a more precise mechanism involved in the effect of $\beta$-catenin signaling or calcineurin-NFAT signaling during an acute phase of pressure overload-induced cardiac hypertrophy.

\section{References}

1. Molkentin JD, Dorn GW. Cytoplasmic signaling pathways that regulate cardiac hypertrophy. Ann Rev Physiol 2001;63: 391-426.

2. Molkentin JD, Lu JR, Antos CL, Markham B, Richardson J, Robbins $\mathbf{J}$, et al. A calcineurin-dependent transcriptional pathway for cardiac hypertrophy. Cell 1998;93:215-28.

3. Antos CL, McKinsey TA, Frey N, Kutschke W, McAnally J, Shelton JM, et al. Activity glycogen synthase-3b suppresses cardiac hypertrophy in vivo. Proc Natl Acad Sci U S A 2002;99:907-12.

4. Shimoyama M, Hayashi D, Takimoto E, Zou Y, Oka T, Uozumi $\mathrm{H}$, et al. Calcineuryn plays a critical role in pressure overload-induced cardiac hypertrophy. Circulation 1999;100: 2449-54.

5. Olsen EN, Schneider MD. Sizing up the heart: development redux in disease. Genes Dev 2003;17:1937-56.

6. Haq S, Choukroun G, Kang ZB, Ranu H, Matsui T, Rosenzweig A, et al. Glycogen synthase kinase-3 $\beta$ is a negative regulator of cardiomyocyte hypertrophy. J Cell Biol 2000; 151:117-29.

7. Haq S, Michael A, Andreucci M, Bhattacharya, Dotto P, Walters B, et al. Stabilization of $\beta$-catenin by a Wntindependent mechanism regulates cardiomyocyte growth. Proc Natl Acad Sci U S A 2003;100:4610-5. 